Collection: COST Action FP0905

"Biosafety of forest transgenic trees and EU policy directives"

Guest Editors: Cristina Vettori, Matthias Fladung

\title{
Next generation biotechnology: how sophisticated constructs lead to further insights and new approaches towards biotechnology's demands
}

\author{
Axel Hinze, Dirk Becker
}

For most plants transformation methods and regeneration protocols are readily available, leading to more complicated and differential research in plant science. The application of expression or RNAi constructs in biotechnology can lead to unwanted effects and detrimental phenotypes, which may cause great problems in the regeneration and breeding of transgenic plants. To circumvent this set back we resort to the transformation of inactive constructs. After the creation and establishment of transgenic lines the construct is specifically activated and the effects on the plants can be studied in detail, even if the resulting phenotype has harmful consequences. The activation of the constructs is carried out by means of induced site-directed recombination. The application of an heat shock leads to the expression of a recombinase, which excises the spacer fragment and leads to the desired effect on the plants. The feasibility of those constructs could be shown for RNAi, induced expression and even marker excision and confinement approaches demonstrating the huge variability of the new construct design.

Keywords: Site-specific Recombination, Cre/loxP, FLP/FRT, Heat Shock Inducible Promoter, Tissue Specific Expression, RNAi

\section{Introduction}

There are several recombinases, which are able to induce changes in the structure of a transgenic sequence in vivo. The most commonly known are the Cre/loxP system of Bacteriophage P1 (Sternberg \& Hamilton 1981), the FLP/FRT system of Saccharomyces cerevisiae (Broach et al. 1982) and the $\mathrm{R} / R S$ system of Zygosaccharomyces rouxii (Araki et al. 1985), although a variety of other usable recombinases exist (Nern et al. 2011). These enzymes belong to the tyrosine recombinase family and have highly specific recognition sites, which compose of palindromic inverted repeats of 12-14 bp and a spacer sequence of 7-8 bp. These enzymes are able to either invert or excise sequences between recognition sites as part of an intramolecular recombination, but also to perform an intermolecular site-directed integration of DNA, depending on the orientation and type of recognition sites. These properties have been reported in several plants (Lyznik et al. 1993 - for an overview, see Wang \& Yau 2011) and are also wildly known in the field of genomic engineering with mammalian and drosophila cells as well as several prokaryotes (Kolb 2002). General considerations for the use of recombinases and the use of variable constructs should be the right choice of promoters, regulating when or where which recombinase should be active (Van Ex et al. 2009). For an effective and reliable recombination, the codon usage and $\mathrm{G} / \mathrm{C}$ content of the gene should be adapted from the host organism to ensure gene expression (Song \& Niederweis 2007). Also detrimental effects of the recombinase like chlorosis, growth aberration and even sterility could be observed in tobacco, petunia and tomato (Coppoolse et al. 2003).

\section{Excision of marker genes and transgene confinement}

In the last twenty years there have been several successful attempts of marker gene excision in a variety of plants (Russell et al. 1992, Dale \& Ow 1992, Kerbach et al. 2005, Darbani et al. 2006, Gidoni et al. 2008). While the first attempts of marker excision were carried out with constitutive promoters, the last five years have brought a shift to a finer regulated on demand activation of the marker excision. For example, the use of either an inducible Drosophila heat shock promoter in potato (Cuellar et al. 2006 - ex- $\square$ Biocenter Klein Flottbek, EBBT, University of Hamburg, Ohnhorststr. 18, D-22609 Hamburg (Germany)

@, Dirk Becker (becker@botanik.unihamburg.de)

Received: Jan 24, 2012 - Accepted: May 23, 2012

Citation: Hinze A, Becker D, 2012. Next generation biotechnology: how sophisticated constructs lead to further insights and new approaches towards biotechnology's demands. iForest 5: 131-137 [online 201206-13] URL: http://www.sisef.it/ iforest/contents?id=ifor0620-005

cision rate up to $14 \%$ ), the salicylic-acid inducible promoter from tobacco in tomato (Ma et al. 2008 - excision efficiency of 41\%) or a tissue specific promoter like NTM19 (microspore specific) from tobacco (Mlynárová et al. 2006 - excision failure rate of $0.024 \%$ ) leads to the activation of the recombinase and the highly effective excision of genes. Due to the general design of these constructs, they are only suited for a specific application or plants which can be held as scions in laboratory dimensions like tobacco or poplar. For breeding and in field application of grassy plants, a different and generally applicable type of constructs is needed. As a new approach, we created constructs which are composed out of two separate units (Fig. 1). One region transfers a certain set of traits to the plant and is used for transgene selection. The second unit controls the heredity of a transgene by seeds. This is achieved through a combination of inducible and germ line specific regulation of recombinase expression. These constructs were introduced into the maize genome, by means of Agrobacterium-mediated transformation. Screening of transgenic lines was performed and seeds were produced from the different lines. After the seeding of $\mathrm{T}_{1}$-plants, the inducible recombinase was activated via heat shock. The expression of the recombinase was monitored through RT-PCR and Western blot (data not shown), while the spacer excision of the recombinase and its promoter (Fig. 1A) was confirmed using Southern blot experiment(Fig. 2). The loss of an intervening sequence between recombination sites for the first recombinase resulted in the reduced size of the hybridization product (Fig. 1B and Fig. 2 lines 288 and 3440). After the removal of the spacer sequences, a tissue specific promoter sequence is brought in front of the second recombinase. In this confinement approach the pollen specific ZmMADS2 promoter (Schreiber et al. 2004) was used, which would lead to an activation of the second recombinase during pollen development. This recombinase excises the 


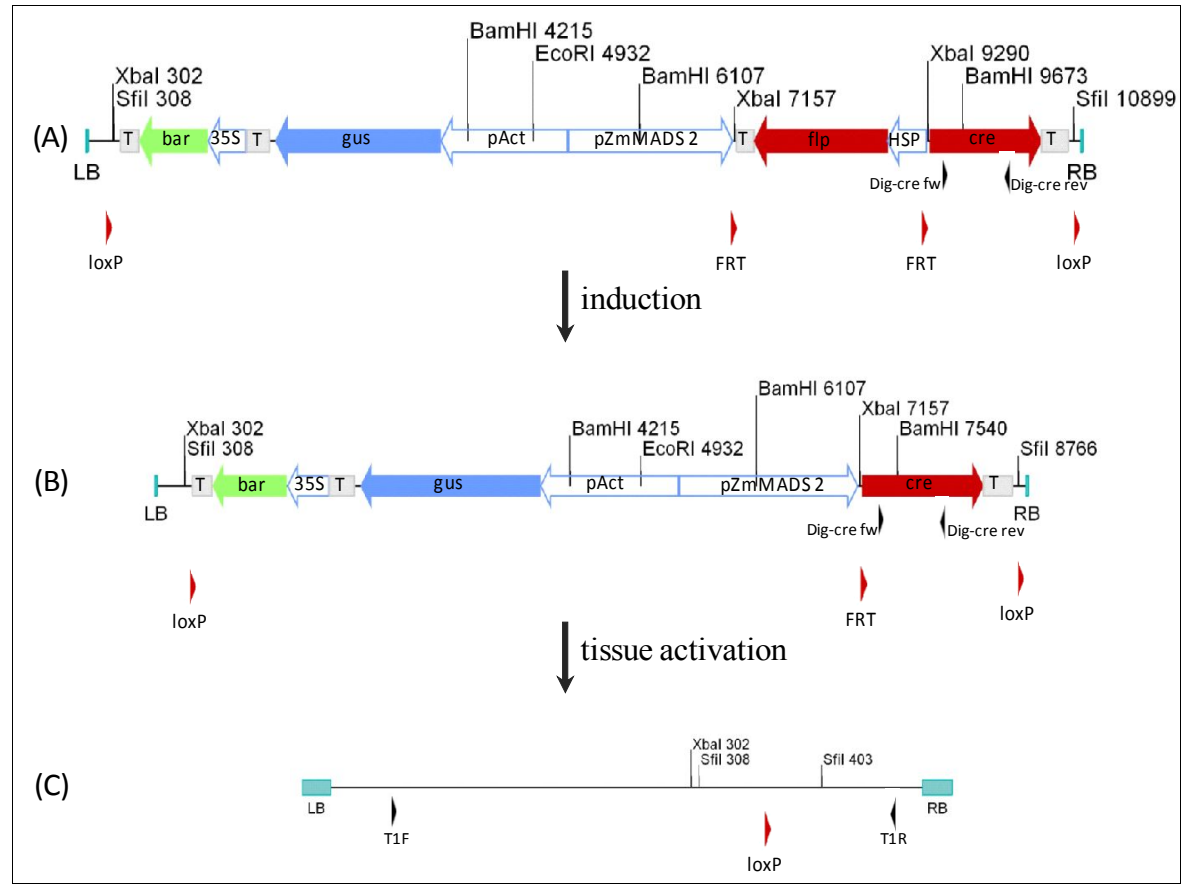

Fig. 1 - Schema of the confinement construct. The construct composes out of two selectable marker genes, bar and uidA, for the selection of transgenic plants and offspring. The excision of the transgenic sequences from the host genome is interrupted by a spacer sequence, which is flanked by FRT sites. The spacer sequence is made of the FLP recombinase and the GmHSP 17.5E promoter from soy bean (Czarnecka et al. 1985), regulating the activity of the FLP recombinase. On the 5' end of the spacer sequence the pollen specific promoter ZmMADS2 is located. Following the spacer sequence on the $3^{\prime}$ side is the Cre recombinase. After the transformation of the dormant construct (A) the activation of the constructs is induced through the application of a heat shock, leading to the expression of the FLP recombinase and the excision of the spacer sequence. This brings the pollen specific promoter in regulating distance to the Cre recombinase (B). After the developmental activation of the ZmMADS2 promoter the Cre recombinase is expressed in pollen. This will excise the whole construct, leaving only one

framing loxP recombinase recognition sites in the host genome, rendering the phenotype of the $\mathrm{T}_{2}$ - progeny wild type (C). The black arrows show relevant primer binding sites, Dig-cre fw and Dig-cre rev, for the construction of a labeled probe for the determination of heat shock activation (A) and (B). The primer binding sites T1F and T1R were used to confirm the excision of the construct (C). (T): terminator sequence (nos/35S); (bar): Phosphinothricin acetyltransferase gene; (gus): uidA gene from E. coli; (pAct): Actl-F promoter from rice; (pZmMADS2): MADS2 promoter from Zea mays; (flp): FLP recombinase; (Stls1): Stls1-Intron from potato; (HSP): GmHSP 17.5E promoter; (35S): CaMV35S promoter; (cre): cre recombinase; (LB) and (RB): Left- and right border sequences; (FRT): FRT recognition sites; (loxP): loxP recognition sites; (Dig cre fw) and (Dig-cre rev): primers.

transgenic sequence, leaving only the insignificant residue of the right and left border sequences from the transformation event and a single recombinase recognition site (Fig. 1C) in the host genome. After the transformation of maize embryos, we were able to obtain 268 supposedly independent lines. 28 lines could be identified as single copy events. After the heat shock application 10 lines showed full excision of the spacer fragment in Southern blot experiments. After crossing the pollen from those lines into the maize inbred line A188, three crossing events showed extreme deviation from the
Mendelian segregation. One line even achieving a transgene excision efficiency of $100 \%$ in the following generation.

To verify the removal of transgenic sequences from the host genome we crossed pollen from fully activated lines into inbred A188 maize lines. Fourteen days after pollination

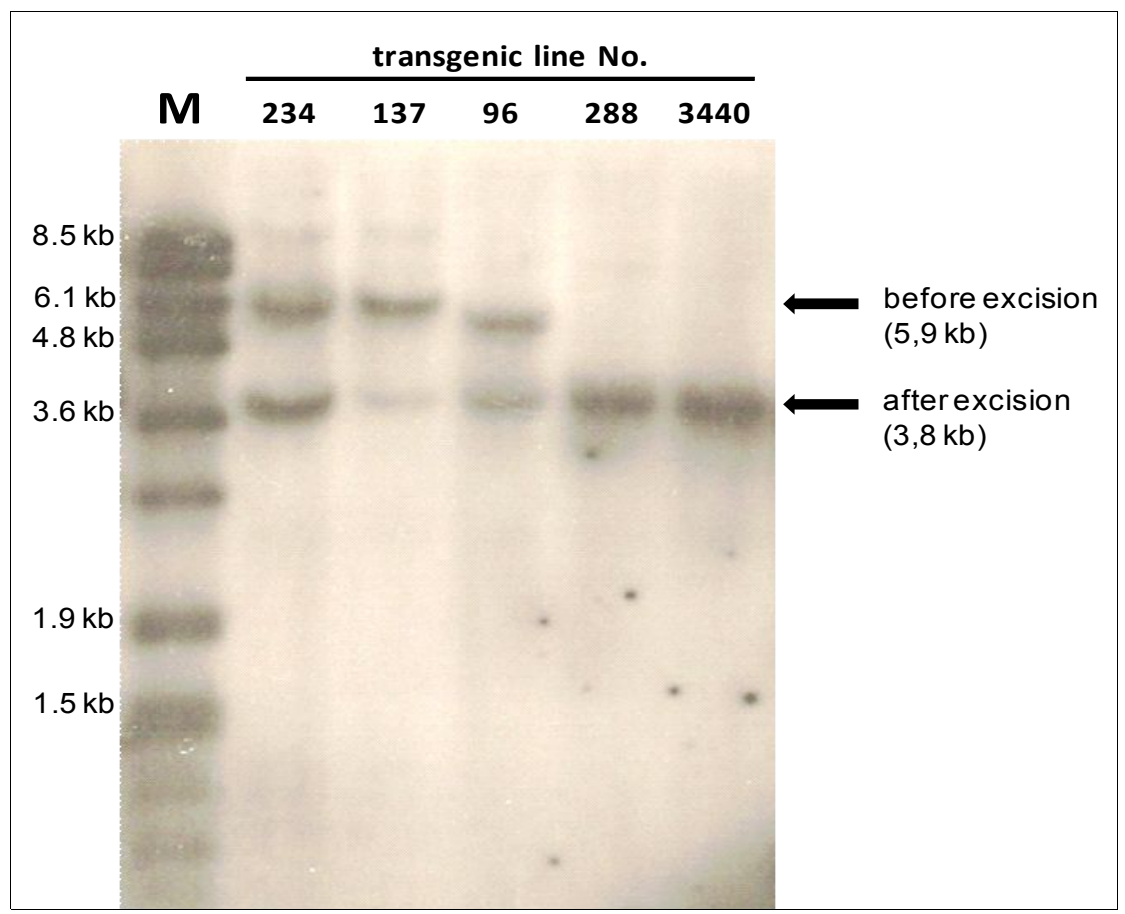

Fig. 2 - Activation of the confinement construct. Southern Blot analysis of the heat shock induced recombination. The excision of the spacer fragment in the construct leads to a specific size reduction in the fragment after SfiI / EcoRI digestion. Here are shown several transgenic lines (No. 234; 137; 96; 288 ; 3440) after an applied heat shock. The expected band for the non-induced transgenic sequence lies at $5.9 \mathrm{~kb}$. After the heat shock, the size drops to $3.8 \mathrm{~kb}$ through the excision of the spacer fragment, composed out of the inducible promoter and the first recombinase. In line 1 the DIG VII marker (Roche) is shown. Lines 2-4 show chimerical plants in the analysis with an only partly excised spacer fragment. Lines 5 and 6 show fully activated plants after the complete excision of the spacer fragment in compliance with the Fig. 1B. 
Fig. 3 - Genetic analysis of non-transgenic phenotype in $T_{1}$ - progeny. After the outcrossing of transgenic maize pollen into inbred line A188, the offspring were histochemically stained for the expression of the marker gene uidA. A normal Mendelian segregation of the transgene would result in a roughly 50:50 segregation in the next generation. From lines with abnormal high amounts of wild type offspring genomic DNA (gDNA) was extracted from non-transgenic phenotype progeny and the excision of the construct verified via PCR. Line 1 and 10: O'GeneRuler ${ }^{\mathrm{TM}} 1 \mathrm{~kb}$ Plus DNA Ladder (Fermentas). Lines 2-9: Verification of transgene excision PCR of gDNA extracted from the progeny plants from Line 288 . Line 11: negative control maize gDNA Line 12: water control without gDNA. Line 4 and 7 show the expected $450 \mathrm{bp}$ excision fragment, in compliance with Fig. 1C. Isolation and sequencing of the products verified the successful excision events.

the kernels were harvested and the immature embryos isolated. After the germination of the embryos in tissue culture the small seedlings were transferred into soil. After a few days histological GUS-staining of leaf tissue was performed (data not shown) to screen for significant deviations from Mendelian inheritance pattern. Genomic DNA was isolated from lines showing a highly significant amount of non-transgenic phenotype offspring $(>70 \%)$ to verify the excision on a molecular basis. For this primers were designed at the borders of the construct (Fig. 1C - black arrows). In case of an excision event a PCR should lead to a $450 \mathrm{bp}$ fragment (Fig. 3), which was isolated and the final excision proven through sequencing.

\section{Cassette exchange and site directed integration}

The integration site of transgenic sequences into the host genome, mediated by Agrobacterium is supposed to be favorably in AT-rich regions, as it was shown for tobacco (Gheysen et al. 1987), Arabidopsis (Mayerhofer et al. 1991), rice (Takano et al. 1997) and poplar (Kumar \& Fladung 2002). Despite this, the expression of genes in those regions may still differ greatly from one transgenic line to another. The insertion of certain traits into a well defined region is already possible in animal cells and fungi (Smithies et al. 1985, Thomas \& Capecchi 1987, Capecchi 1989, Hinnen et al. 1978, De Lozanne \& Spudich 1987), due to their ability of homologous recombination. A highly effective method for the targeted integration of transgenic sequences via homologous recombination into the genome of higher plants is not yet available, although a lot of research has been committed (Paszkowski et al. 1988, Puchta et al. 1995, Salomon \& Puchta 1998). An available alternative is the

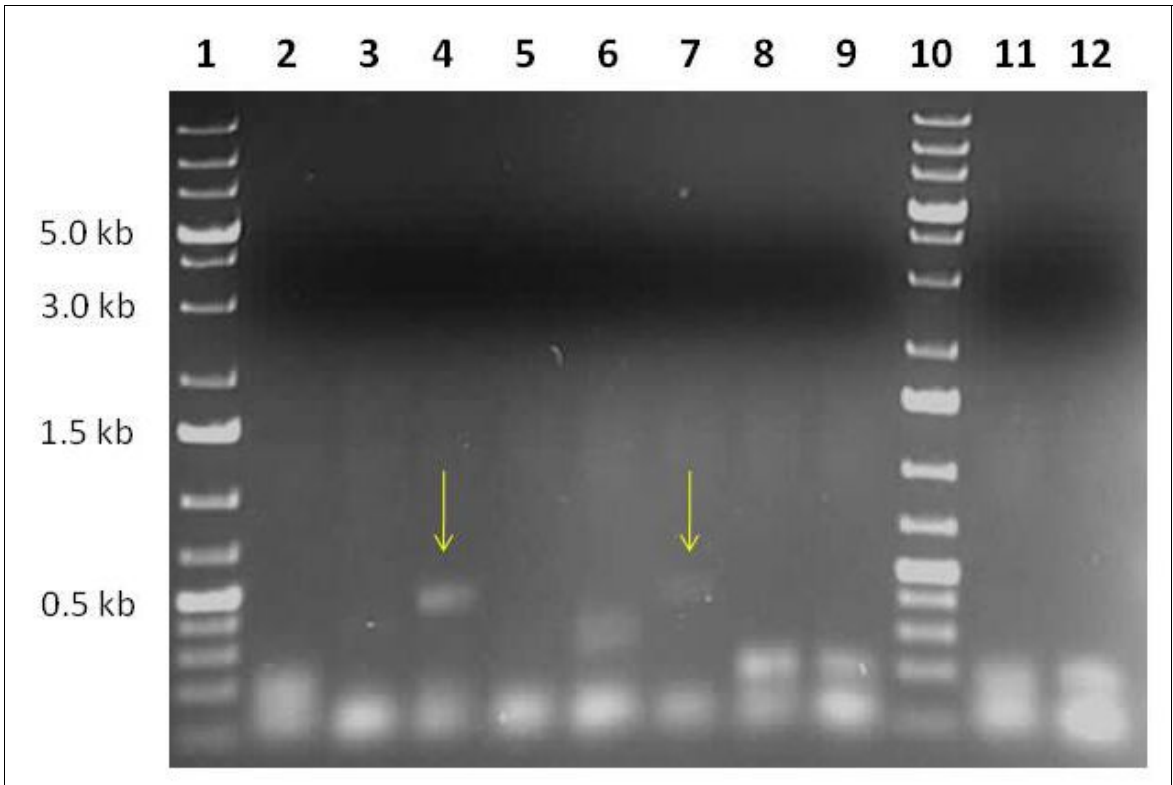

site-specific integration of transgenic se- achieve this, double transgenic poplar lines quences or cassette exchange via recombina- were developed carrying one transgenic setion (Vergunst \& Hooykaas 1998, Araki et quence with an inducible FLP recombinase al. 2002, Fladung \& Becker 2011). To (Fig. 4A) and a second integration event

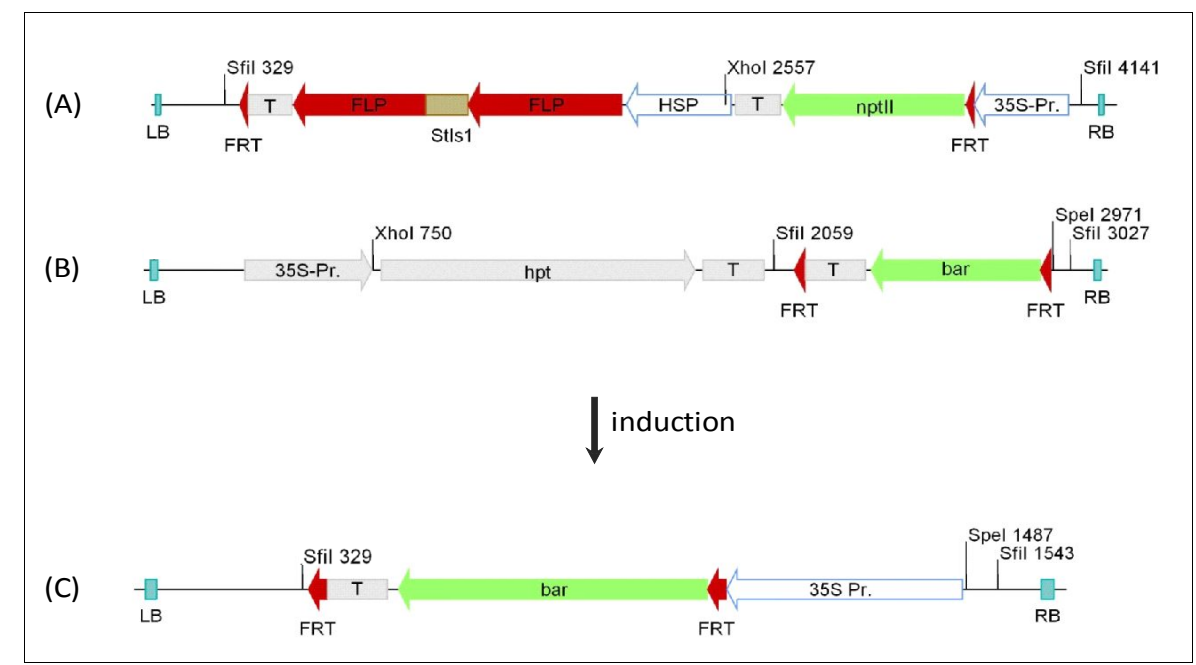

Fig. 4 - Construct schema of the cassette exchange experiments. Schematic representation of the integrated acceptor (4A) and donor (4B) construct used for site directed cassette exchange in poplar and the integration site after the successful site directed integration (4C). The targeted integration through cassette exchange was carried out with double transgenic lines. The integrated acceptor construct carries the selection marker nptII and the FLP recombinase from yeast. The expression of the FLP is under control of the Gmhsp 17.5E promoter from soybean. Collateral orientated FRT- sites are flanking the 3` end of the FLP terminator sequence and the $5^{\prime}$ end of the selection marker nptII between gene and promoter. A $35 \mathrm{~S}$ promoter is located on the $3^{\prime}$ end outside the recombination sites. In the inactivated state, the $35 \mathrm{~S}$ promoter drives the nptII gene. The second insertion carries a promoterless bar gene, flanked by parallel FRT sites and an additional selective marker gene hpt. After the heat activation the expression of the FLP recombinase leads to the excision of the FRT flanked regions in the constructs. Through the possibility of site directed integration the promotorless bar gene can be inserted in the acceptor construct (4A) in front of the $35 \mathrm{~S}$ promoter leading to a functional selective marker gene (4C). (T): terminator sequence (nos/35S); (bar): Phosphinothricin acetyltransferase gene; (FLP): FLP recombinase; (Stls1): Stls1-Intron from potato; (HSP): GmHSP 17.5E promoter; (nptII): Neomycin phosphotransferase gene; (35SPr., 35S): CaMV35S promoter; (hpt): Hygromycin phosphotransferase gene; (LB) and (RB): Left- and right border sequences; (FRT): FRT sites. 

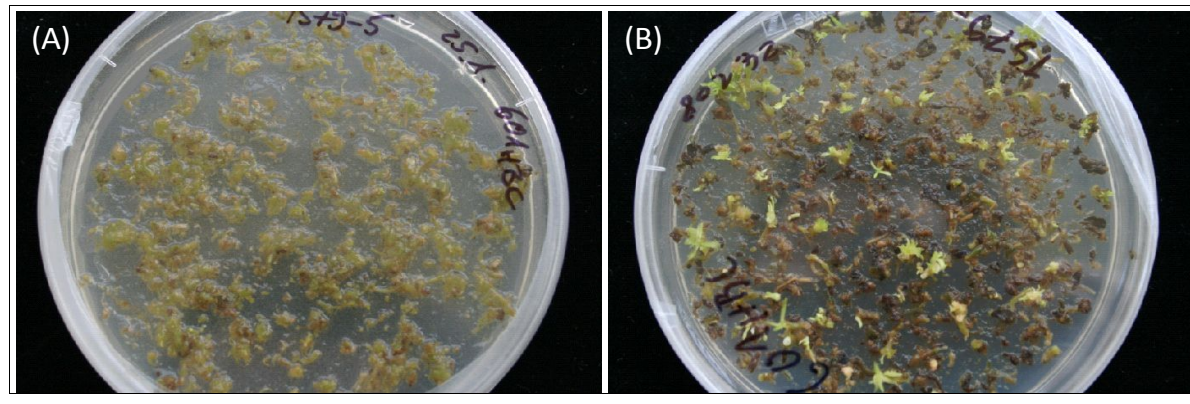

with the target sequence (Fig. 4B). After the transformation, the cassette exchange was induced via heat shock treatment, the leaf tissue crushed and the regenerating calli were screened for successful expression of the target gene (Fig. 4C). The site-directed cassette exchange led to the expression of a hygromycin resistance marker gene, which could be readily selected on regeneration media in a frequency of up to $7.4 \%$ (Fig. 5 - Fladung et al. 2010). Higher cassette exchange frequencies of up to $44 \%$ could be shown in Arabidopsis using the Cre/loxP system (Louwerse et al. 2007). With the possibility of a site-directed integration or recombinase mediated cassette exchange one can circumvent the extensive work of determining high expression lines for every new construct. After the construction of an acceptor line, with a thoroughly characterized integration site, new constructs could be precisely inserted through site-directed insertion or cassette exchange mediated by a site-specific recom-

bination. The expression of a new construct could be easily predicted and would not be subject to great variability. This could be carried out through the construction of new transgenic lines, which would be crossed into the acceptor line. The transgene transmission could be realized in the resulting hybrid plants and genetically purified through back crosses. Another alternative would be the transient or stable retransformation of the acceptor line with the integration construct, followed by a cassette exchange.

\section{Inducible gene expression and induced RNAi in plants}

\section{Induced gene expression}

Tissue specific expression of genes for the accumulation of certain products has been successfully used in different plants. For example the production of resveratrol, which is supposed to have beneficial effects on human health, has been carried out in barley

(A)

Fig. 6 - Schema of inducible expression construct. The construct has the bar marker gene under control of the $35 \mathrm{~S}$ promoter. The expression of the ZmPox-GFP fusion protein triggered by the Ubi-int promoter (ZmUbiquitine promoter with first intron) is interrupted by the GmHSP 17.5E - Cre cassette (A). After the induction of the Cre recombinase via heat shock the spacer will be excised. This will put the constitutive promoter in front of the fusion protein leading to its expression (B). (T): terminator sequence (nos/35S); (bar): Phosphinothricin acetyltransferase gene; (HSP): GmHSP 17.5E promoter; (35S): CaMV35S promoter; (Ubi-Int): Ubiquitin promoter from Zea mays; (ZmPox): Peroxidase gene from Zea mays; (GFP): green fluorescent protein gene; (Cre): cre recombinase; (LB) and (RB): Left- and right border sequences; (FRT): FRT recognition sites; (loxP): loxP recognition sites.
Fig. 5 - Cassette exchange in poplar regenerants. Proof of concept work showing the expression of a hygromycin resistance after a cassette exchange in poplar. Double transgenic lines without inducing treatment showed no regeneration on selective media (A), while induced lines were able to regenerate plantlets (B) in accordance to Fig. 4C.

(Leckband \& Lörz 1998) and in oilseed rape (Hüsken et al. 2005). But the conditional expression of certain substances can lead to unexpected effects on the development of the plants, due to substrate competition and physiological strain (Fischer et al. 1997). To circumvent such effects, especially when the expression is supposed to take place in the reproductive organs of the plant, the transformation can be carried out with an inactivated construct (Fig. 6A). This will only transfer an active selection marker resulting in minimal physiological strain for the plant during the breeding process. After the successful breeding, the activation of the construct (Fig. 6B) will lead to the conditional expression of the desired product, while possible negative side-effects can be ignored. The first successful induced expression experiments have been carried out, with a gfpsequence fused to a maize-peroxidase gene. To verify the feasibility of the constructs, maize embryos 14 days after pollination were transiently transformed through particle bombardment. After the transformation the embryos showed no GFP-signal (Fig. 7A). The induction of the constructs leads to the excision of the spacer region followed by an expression of the fusion protein shown as GFP-signals in transformed cells (Fig. 7B). These data demonstrated the viability of this approach for conditional protein expression in plants.

\section{Induced $R N A i$}

RNAi has been used widely and successfully for genome analysis in several different plants. In most constructs a promoter is followed by a sense and antisense sequence of the gene of interest, devided by a spacer or an intron (Waterhouse et al. 1998, Smith et al. 2000). Due to the large homologies in the vector, sequence cloning and construction can be quite complicated. Efforts have been made to make the cloning of RNAi-constructs easier for high throughput application (Wesley et al. 2001), but still the issue of homologue sequences remains unsolved and can be quite troublesome. Furthermore, can the construction of mRNA-RNAi libraries be hindered by the development of lethal effects or phenotypes in the plants. To overcome all those limitations on RNAi, our group has adapted the opposing-dual promoter system 
Fig. 7 - Transiently induced gene expression as proof of concept. Validation of the indu-

cible expression. 14 days after pollination maize scutellum underwent heat shock treatexpression constructs (Fig. 6A). Left: scutellar tissue without induction, right: tissue 24 hours after a heat shock treatment, showing the expression of a GFP-peroxidase fusion protein. ment after biolistic transformation with the
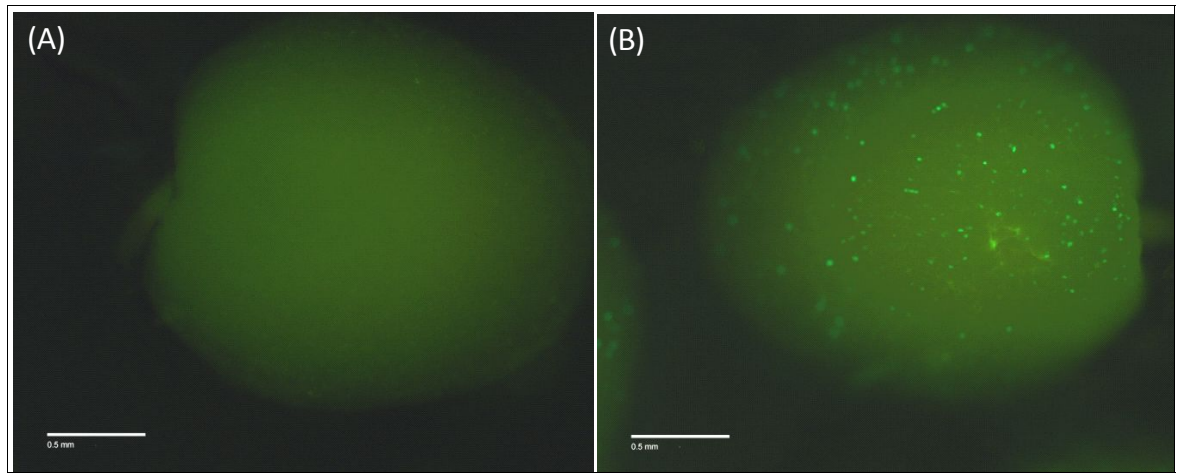

from fungi (Mette et al. 2000, Schmidt et al. 2012) for easy cloning. To circumvent lethal or detrimental RNAi-effects the transformations are carried out with inactivated constructs (Fig. 8A). After the successful transformation, the RNAi constructs are specifically activated through a heat shock, which will excise a spacer fragment (Fig. 8B). After the excision of the spacer a constitutive promoter will be in front of the second recombinase. This leads to the expression of the second recombinase, which will deliver the complement promoter in activation range to the RNAi sequences (Fig. 8C). The expression of complementary RNA strands initiated through both promoters results in the formation of double stranded RNA, which will induce the RNAi-effect. The feasibility of the opposing promoter system for RNAi could be shown for tobacco (Schmidt et al. 2012) and poplar (Fladung M, personal communication).

\section{Conclusions}

The use of recombinases in our constructs has facilitated a variety of new possibilities. The transformation of inactivated constructs leads to the real autonomy of the transformation process and the following transgene effect on the plant, in contrast to tissue specific or conditional expression. After the transformation of plant material transgenic lines can be bred and seeds obtained, containing a dormant construct. This separation allows researchers to induce certain traits in the plants and study the following effects without concerns for the viability of the transgenic line or the risk of destroying valuable seed material. This is of special interest for the field scale application as a confinement strategy, where the breeding and crossing of transgenic sequences into different lines is necessary, but stands in harsh contrast to the need for a reliable and highly effective excision during the germ-line development. Another great advantage arises for basic studies in plants. The simple but effective cloning strategy enables researchers to create whole mRNA-RNAi libraries, where potentially lethal RNAi effects can be transformed and bred. The effects of the RNAi can be studied in detail and at any time during development after the activation of the constructs. The same applies for the induced expression of proteins. The conditional expression of certain proteins leads to an enormous physiolo- gical strain and might have a negative effect on the plants in general or the fertility of the expression lines. Furthermore, will the construction of so called acceptor lines enable the researcher to circumvent the laborious

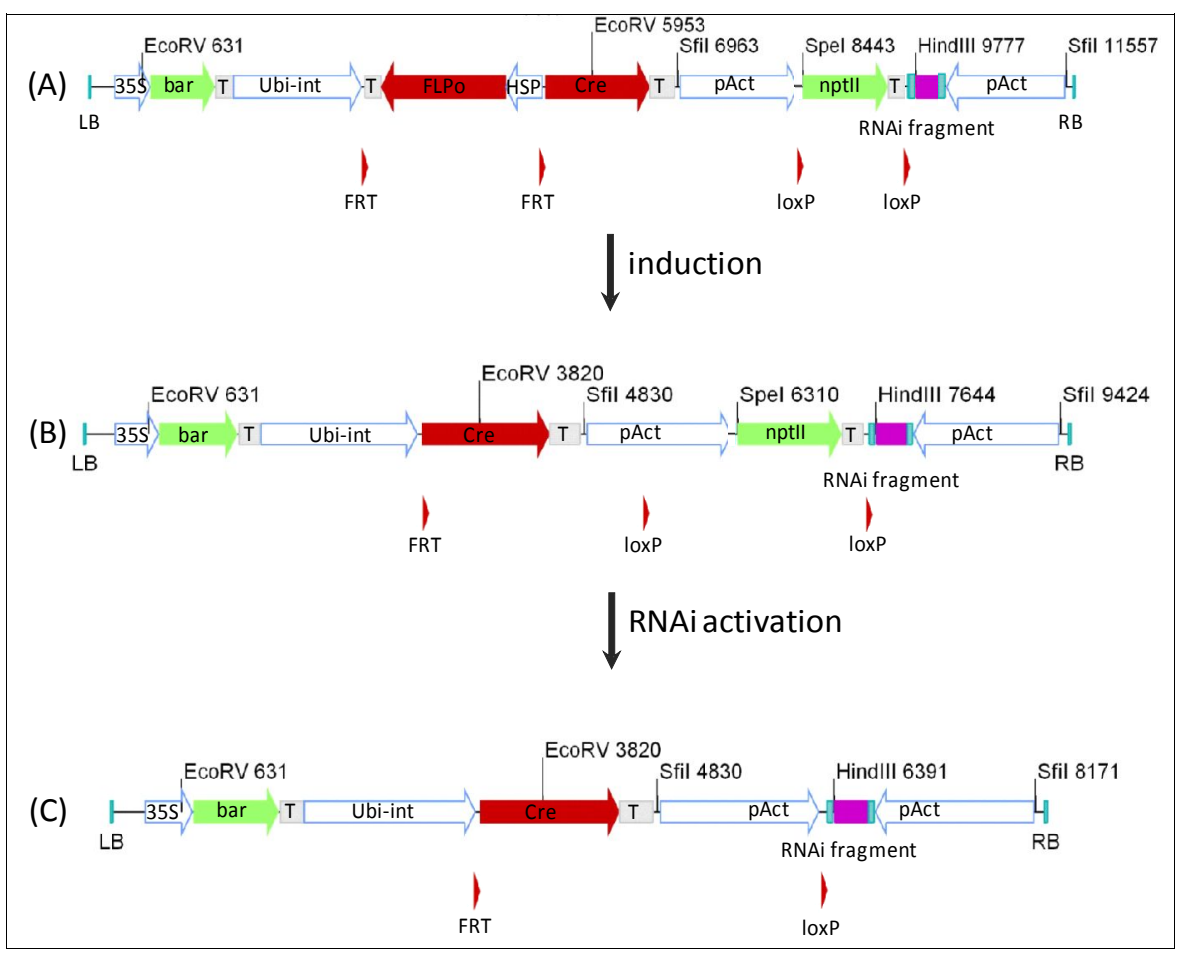

Fig. 8 - Inducible RNAi construct schema. The construct has a bar gene regulated by a 35S promoter for general selection. With the nptII gene, regulated by the pAct, it is possible to select against premature activation of the RNAi during tissue culture. The nptII fragment, flanked by loxP sites, is also used as an inactivating spacer for the antisense stand of the RNAi sequence. Furthermore lies an inverted pAct promoter at the 3 'end of the construct, producing a sense stand of the RNAi fragment. For the activation of the construct a heat shock will lead to the expression of the FLP, excising itself and its promoter, bringing the Ubi-int promoter in regulating distance to the cre gene (B). After the first induction, the expression of the cre recombinase leads to the excision of the nptII marker gene, putting its pAct promoter on the other side of the RNAi fragment, leading to the expression of the antisense strand. The production of complementary RNA strands leads to the formation of double stranded RNA, resulting in the initiation of the RNAi effect. (T): terminator sequence (nos/35S); (bar): Phosphinothricin acetyltransferase gene; (gus): uidA gene from E. coli; (Ubi-Int): Ubiquitin promoter from Zea mays; (pAct): Actl-F promoter from rice; (pZmMADS2): MADS2 promoter from Zea mays; (FLPo): Codon optimized FLP recombinase; (HSP): GmHSP 17.5E promoter; (35S): CaMV35S promoter; (Cre): Cre recombinase; (nptII): Neomycin phosphotransferase gene; (LB) and (RB): Left- and right border sequences; (FRT): FRT recognition sites; (loxP): loxP recognition sites. 
search for high expression transformation events. This leads to an acceptable and reliable expression of the integrated sequence.

\section{Outlook}

The major setback of the inducible constructs is the lacking of non-leaky and reliable inducible system which performs in field scale applications. While a set of inducible promoters are available, the majority are only suited for scientific approaches. Inducible promoters either require the application of chemicals, which might be scorned at in field trials or depend on a certain physiological strain, which imposes problems in large scale impulse response. Furthermore, most inducible promoters are activated by a variety of factors. For several heat shock promoters it was shown that a variety of impulses, such as heavy-metal levels, were able to induce an expression. This instability will pose problems in the premature or improper activation during field application depending on the environmental circumstances. On the other hand, the large scale application of hormone or chemical solutions is not very accurate and the achievement of inducing concentration might be compromised, not considering environmental or public health concerns. The answer might lie in the development of synthetic promoters or the adaption of bacterial promoters for the use in plants. The most desired traits for such an inducible promoter are the specificity and irrevocability of the signal. It is supposed to be exclusive, easy to impose and should lead to a high gene expression without severe harm for the plant. Another future aspect of recombinase depending approaches is the search for new reliable enzymes. There have been a lot of new findings and developments on this field, clearly showing that some of the recombinases are not suited for every host system. High expression levels of the Cre-recombinase leads to malformation in Drosophila optical cells and is supposed to have pseudo-recognition sites in the murine and human genome. Therefore the variety of different recombinases which are available should be thoroughly tested for their applicability in the possible host organisms. Even enzymes with a very low activity but high specificity can be adapted for different plants after optimization of codon usage and adaptation on the host. The variety and exclusiveness of recombinases throughout the different species, as well as the modification and adaptability of their recognition sites, turn recombinases into a powerful tool in next generation biotechnology of plants. Consequentially, new and more complex constructs will be derived with the different possibilities of integration, inverting and excision all carried out in one construct. This gives rise to highly complex constructs, which will lead to a tightly monitored and predictable transgene expression, not following the unchecked expression design of previous vectors.

\section{Acknowledgments}

We would like to thank Nadja Schmidt, Benjamin Möller and Olaf Pollak for providing exemplary pictures from their ongoing work, as well as François Perrineau for helpful comments on the manuscript. Furthermore we would like to thank Matthias Fladung for the invitation to the COST meeting in Hamburg 2011 and DNA Cloning Services, Hamburg for the cloning of the constructs.

This work has been presented at the "First Biosafety Workshop" of COST Action FP0905, held in Hamburg (Germany) on September $9^{\text {th }}, 2010$.

\section{References}

Araki H, Jearnpipatkul A, Tatsumi H, Sakurai T, Ushio K, Muta T, Oshima Y (1985). Site-specific recombinase, $\mathrm{R}$, encoded by yeast plasmid pSR1. Journal of Molecular Biology 225: 25-37. - doi: 10.1016/0022-2836(92)91023-I

Araki K, Araki M, Yamamura KI (2002). Site-directed integration of the cre gene mediated by Cre recombinase using a combination of mutant lox sites. Nucleic Acids Research 30 (19): e103. - doi: 10.1093/nar/gnf102

Broach JR, Guarascio VR, Jayaram M (1982). Recombination within the yeast plasmid $2 \mathrm{mu}$ circle is site-specific. Cell 29(1): 227-234. - doi: 10.1016/0092-8674(82)90107-6

Capecchi MR (1989). Altering the genome by homologous recombination. Science 244 (4910): 1288-1292. - doi: 10.1126/science. 2660260

Coppoolse ER, de Vroomen MJ, Roelofs D, Smit J, van Gennip F, Hersmus BJM, Nijkamp HJJ, van Haaren MJJ (2003). Cre recombinase expression can result in phenotypic aberrations in plants. Plant Molecular Biology 51: 263-279. doi: 10.1023/A:1021174726070

Cuellar W, Gaudin A, Solórzano D, Casas A, Nopo L, Chudalayandi P, Medrano G, Kreuze J, Ghislain M (2006). Self-excision of the antibiotic resistance gene nptII using a heat inducible Cre-loxP system from transgenic potato. Plant Molecular Biology 62 (1-2): 71-82. - doi: 10.1007/s11103-006-9004-3

Czarnecka E, Gurley WB, Nagao, RT, Mosquera LA, Key JL (1985). DNA sequence and transcript mapping of a soybean gene encoding a small heat shock protein. Proceedings of the National Academy of Sciences USA 82: 37263730. - doi: 10.1073/pnas.82.11.3726

Dale EC, Ow DW (1992). Gene transfer with subsequent removal of the selection gene from the host genome. Proceedings of the National Academy of Sciences USA 88 (23): 1055810562. - doi: 10.1073/pnas.88.23.10558

Darbani B, Eimanifar A, Stewart CN, Camargo WN (2006). Methods to produce marker-free transgenic plants. Biotechnology Journal 2 (1): 83-90. - doi: 10.1002/biot.200600182
De Lozanne A, Spudich JA (1987). Disruption of the Dictyostelium myosin heavy chain gene by homologous recombination. Science 236 (4805): 1086-1091. - doi: 10.1126/science. 3576222

Fischer R, Budde I, Hain R (1997). Stilbene synthase gene expression causes changes in flower colour and male sterility in tobacco. The Plant Journal 11 (3): 489-498. - doi: 10.1046/j.1365313X.1997.11030489.x

Fladung M, Becker D (2011). Targeted integration and removal of transgenes in hybrid aspen (Populus tremula $\mathrm{L} . \mathrm{x}$ P. tremuloides Michx.) using site-specific recombination systems. Plant Biology 12 (2): 334-40 Review. Erratum in: Plant Biology 2011 Jan 13 (1): 223. - doi: 10.1111/ j.1438-8677.2009.00293.x

Fladung M, Schenk TMH, Polak O, Becker D (2010). Elimination of marker genes and targeted integration via FLP/FRT recombination system from yeast in hybrid aspen (Populus tremula $\mathrm{L}$. $\times P$. tremuloides Michx.). Tree Genetics and Genomes 6 (2): 205-217. - doi: 10.1007/s11295009-0241-x

Gheysen G, Van Montagu M, Zambryski P (1987). Integration of Agrobacterium tumefaciens transfer DNA (T-DNA) involves rearrangements of target plant DNA. Proceedings of the National Academy of Sciences USA 84: 61696173. - doi: 10.1073/pnas.84.17.6169

Gidoni D, Srivastava V, Carmi N (2008). Sitespecific excisional recombination strategies for elimination of undesirable transgenes from crop plants. In Vitro Cellular and Developmental Biology - Plant 44 (6): 457-467. - doi: 10.1007/ s11627-008-9140-3

Hinnen A, Hicks JB, Fink GR (1978). Transformation of yeast. Proceedings of the National Academy of Sciences USA 75 (4): 1929-1933. doi: 10.1073/pnas.75.4.1929

Hüsken A, Baumert A, Milkowski C, Becker HC, Strack D, Möllers C (2005). Resveratrol glucoside (Piceid) synthesis in seeds of transgenic oilseed rape (Brassica napus L.). Theoretically Applied Genetics 111 (8): 1553-1562. - doi: 10.1007/s00122-005-0085-1

Kerbach S, Lörz H, Becker D (2005). Site-specific recombination in Zea mays. Theoretically Applied Genetics 111 (8): 1608-1616. - doi: 10.1007/s00122-005-0092-2

Kolb AF (2002). Genome engeneering using sitespecific recombinases. Cloning and stem cells 4 (1): 65-80 - doi: 10.1089/153623002753632066 Kumar S, Fladung M (2002). Transgene integration in aspen: structures of integration sites and mechanism of T-DNA integration. Plant Journal 31 (4): 543-451. - doi: 10.1046/j.1365-313X 2002.01368.x

Leckband G, Lörz H (1998). Transformation and expression of a stilbene synthase gene of Vitis vinifera L. in barley and wheat for increased fungal resistance. Theoretically Applied Genetics 96: 1004-1012. - doi: 10.1007/s001220050832

Louwerse JD, van Lier MC, van der Steen DM, de Vlaam CM, Hooykaas PJ, Vergunst AC (2007). Stable recombinase-mediated cassette exchange in Arabidopsis using Agrobacterium tumefa- 
ciens. Plant Physiology 145: 1282-1293. - doi: 10.1104/pp.107.108092

Lyznik LA, Mitchell JC, Hirayama L, Hodges TK (1993). Activity of yeast FLP recombinase in maize and rice protoplasts. Nucleic Acid Research 21 (4): 969-975. - doi: 10.1093/nar/21. 4.969

Ma BG, Duan XY, Niu JX, Ma C, Hao QN, Zhang LX, Zhang HP (2008). Expression of stilbene synthase gene in transgenic tomato using salicylic acid-inducible Cre/loxP recombination system with self-excision of selectable marker. Biotechnology Letters 31 (1): 163-169. - doi 10.1007/s10529-008-9843-x

Mayerhofer R, Koncz-Kalman Z, Nawrath C, Bakkeren G, Crameri A, Angelis K, Redei GP, Schell J, Hohn B, Koncz C (1991). T-DNA integration: a mode of illegitimate recombination in plants. The EMBO Journal 10 (3): 697-704. [online] URL: http://www.ncbi.nlm.nih.gov/ pmc/articles/PMC452704/

Mette MF, Aufsatz W, van der Winden J, Matzke MA, Matzke AJ (2000). Transcriptional silencing and promoter methylation triggered by double-stranded RNA. The EMBO Journal 19 (19): 5194-5201. - doi: 10.1093/emboj/19.19. 5194

Mlynárová L, Conner A.J, Nap JP (2006). Directed microspore-specific recombination of transgenic alleles to prevent pollen-mediated transmission of transgenes. Plant Biotechnology Journal 4: 445-452. - doi: 10.1111/j.1467-7652. 2006.00194.x

Nern A, Pfeiffer BD, Svoboda K, Rubin GM (2011). Multiple new site-specific recombinases for use in manipulating animal genomes. Proceedings of the National Academy of Sciences USA 108 (34): 14198-14203. - doi: 10.1073/ pnas. 1111704108

Paszkowski J, Baur M, Bogucki A, Potrykus (1988). Gene targeting in plants. The EMBO Journal 7 (13): 4021-4028. [online] URL:
http://www.ncbi.nlm.nih.gov/pmc/articles/PMC455109/

Puchta H, Swoboda P, Gal S, Blot M, Hohn B (1995). Somatic intrachromosomal homologous recombination events in populations of plant siblings. Plant Molecular Biology 28 (2): 281-292. doi: 10.1007/BF00020247

Russell SH, Hoopes JL, Odell JT (1992). Directed excision of a transgene from the plant genome. Molecular and General Genetics 234 (1): 49-59. [online] URL: http://www.springerlink.com/content/r82312h170015203/

Salomon S, Puchta H (1998). Capture of genomic and T-DNA sequences during double-strand break repair in somatic plant cells. The EMBO Journal 17 (20): 6086-6095. - doi: 10.1093/emboj/17.20.6086

Schmidt N, Merker M, Becker D (2012). Novel high-throughput RNAi vectors for plant biotechnology. Plant Breeding (early view) - doi: 10.1111/j.1439-0523.2012.01953.x

Schreiber DN, Bantin J, Dresselhaus T (2004). The MADS box transcription factor ZmMADS2 is required for anther and pollen maturation in maize and accumulates in apoptotic bodies during anther dehiscence. Plant physiology 134 : 1069-1079. - doi: 10.1104/pp.103.030577

Smith NA, Singh SP, Wang MB, Stoutjesdijk PA, Green AG, Waterhouse PM (2000). Total silencing by intron-spliced hairpin RNAs. Nature 407 (6802): 319-320. - doi: 10.1038/35036500

Smithies O, Gregg RG, Boggs SS, Koralewski MA, Kucherlapati RS (1985). Insertion of DNA sequences into human by homologous recombination. Nature 317 (6034): 230-234. - doi: $10.1038 / 317230 \mathrm{a} 0$

Song H, Niederweis M (2007). Functional expression of the Flp recombinase in Mycobacterium bovis BCG. Gene 399 (2): 112-119. - doi: 10.1016/j.gene.2007.05.005

Sternberg N, Hamilton D (1981). Bacteriophage P1 site-specific recombination: I. Recombination between loxP sites. Journal of Molecular Biology 150 (4): 467-486. - doi: 10.1016/0022-2836(81) 90375-2

Takano M, Egawa H, Ikeda JE and Wakasa $\mathrm{K}$ (1997). The structures of integration sites in transgenic rice. Plant Journal 11 (3): 353-361. doi: 10.1046/j.1365-313X.1997.11030353.x

Thomas KR, Capecchi MR (1987). Site-directed mutagenesis by gene targeting in mouse embryoderived stem cells. Cell 51 (3): 503-512. - doi: 10.1016/0092-8674(87)90646-5

Van Ex F, Verweire D, Claeys M, Depicker A, Angenon G (2009). Evaluation of seven promoters to achieve germline directed Cre-lox recombination in Arabidopsis thaliana. Plant Cell Report 28 (10): 1509-1520. - doi: 10.1007/ s00299-009-0750-y

Vergunst AC, Hooykaas PJ (1998). Cre/lox-mediated site-specific integration of Agrobacterium T-DNA in Arabidopsis thaliana by transient expression of Cre. Plant Molecular Biology 38: 393-406. - doi: 10.1023/A:1006024500008

Wang Y, Yau YY (2011). Recombinase technology: alications and possibilities. Plant Cell Report 30: 267-285. - doi: 10.1007/s00299-0100938-1

Waterhouse PM, Graham MW, Wang MB (1998). Virus resistance and gene silencing in plants can be induced by simultaneous expression of sense and antisense RNA. Proceedings of the National Academy of Sciences USA 95 (23): 1395913964. - doi: 10.1073/pnas.95.23.13959

Wesley SV, Helliwell CA, Smith NA, Wang MB, Rouse DT, Liu Q, Gooding PS, Singh SP, Abbott D, Stoutjesdijk PA, Robinson SP, Gleave AP, Green AG, Waterhouse PM (2001). Construct design for efficient, effective and high-throughput gene silencing in plants. Plant Journal 27 (6): 581-590. - doi: 10.1046/j.1365-313X.2001. 01105. $\mathrm{x}$ 